UNIL-IPT-00-08

hep-th/0004014

April 2000

\title{
Localizing Gravity on a String-Like Defect in Six Dimensions
}

\author{
Tony Gherghetta $\rrbracket^{\natural}$ and Mikhail Shaposhnikovfُ \\ Institute of Theoretical Physics \\ University of Lausanne \\ CH-1015 Lausanne, Switzerland
}

\begin{abstract}
We present a metric solution in six dimensions where gravity is localized on a fourdimensional singular string-like defect. The corrections to four-dimensional gravity from the bulk continuum modes are suppressed by $\mathcal{O}\left(1 / r^{3}\right)$. No tuning of the bulk cosmological constant to the brane tension is required in order to cancel the four-dimensional cosmological constant.
\end{abstract}

\footnotetext{
${ }^{1}$ Email: tony.gherghetta@ipt.unil.ch

${ }^{2}$ Email: mikhail.shaposhnikov@ipt.unil.ch
} 
It is an old idea that spacetime may have more than four dimensions, with extra coordinates being unobservable at available energies. A first possibility arises in Kaluza-Klein type theories (see e.g [1] and references therein), where the D-dimensional metric has the form

$$
d s^{2}=g_{\mu \nu}\left(x^{\mu}\right) d x^{\mu} d x^{\nu}-\gamma_{a b}\left(x^{a}\right) d x^{a} d x^{b} .
$$

Here $g_{\mu \nu}$ is the metric of our four-dimensional world, while $\gamma_{a b}$ is the metric associated with $D-4$ (small, with a size $M^{-1}$ ) compact extra dimensions. The compactness of extra dimensions makes them unobservable at energies $E<M$, and manifests itself in the existence of an infinite tower of states with four-dimensional masses $\sim M$.

In fact, the Kaluza-Klein metric is not the most general metric consistent with Poincare invariance in four dimensions. Its generalization was proposed in [2], and is given by

$$
d s^{2}=\sigma\left(x^{a}\right) g_{\mu \nu}\left(x^{\mu}\right) d x^{\mu} d x^{\nu}-\gamma_{a b}\left(x^{a}\right) d x^{a} d x^{b} .
$$

where $\sigma\left(x^{a}\right)$ is a conformal factor depending on the extra coordinates only. A number of specific solutions of the Einstein equations in six-dimensional (6d) spacetime with a positive $6 \mathrm{~d}$ cosmological constant were found in [2], leading to non-compact extra dimensions while still leaving them unobservable at low energies.

Yet another idea leading to non-compact extra dimensions was suggested in [3, 4, 5]. In this case the four dimensions of our world were identified with the internal space of topological defects residing in a higher-dimensional spacetime (e.g. a domain wall in 5d, string in 6d, monopole in $7 \mathrm{~d}$, instanton in $8 \mathrm{~d}$, etc). In these type of backgrounds, as a rule, there are fermionic and scalar zero modes, that can be associated with the four-dimensional particles that we observe. At that time it was not clear how to localize the gauge fields and gravity on topological defects in order to make the whole construction realistic.

The solitons of string theory - D-branes - provide a natural framework for the localization of gauge and matter fields on the worldvolume of the branes [6]. In field theory language the branes can be associated with topological defects. Moreover, in Ref. [7] it was discovered that gravity could be localized on the 3 -brane domain wall in 5 d spacetime. A normalizable graviton zero mode residing on the brane correctly reproduces $4 \mathrm{~d}$ gravity, while the continuum spectrum of $5 \mathrm{~d}$ gravitons living in the bulk, gives only a small correction $\mathcal{O}\left(1 / r^{2}\right)$ to Newton's law at large distances [7]. The metric of the corresponding $5 \mathrm{~d}$ spacetime has the general structure of eq. (2).

The aim of the present paper is to see what happens with gravity around a 3-brane of a specific structure (local string defect in field theory language) in 6d spacetime with a negative cosmological constant. In fact, a regular solution of the Einstein equations in this case for an empty space follows immediately from [2], but does not give any possibility of compactification. However, the existence of a brane with positive tension changes the situation and we find a solution which is very similar to that of Ref. [7]. In contrast to $5 \mathrm{~d}$ case, there is no fine-tuning of the cosmological constant in the bulk to the tension of the brane (the origin of this difference is that the $1 \mathrm{~d}$ space in the domain wall scenario is flat, while the $2 \mathrm{~d}$ space around the string defect can be curved). Similarly to the solution in Ref. [7], there is a normalizible graviton zero mode 
attached to the string-like defect, and the contribution of bulk gravitons is suppressed, leading to $\mathcal{O}\left(1 / r^{3}\right)$ violations of Newton's law. A hierarchy between the four-dimensional Planck scale and the Planck scale in $6 \mathrm{~d}$ can be obtained, leading to a solution of the gauge hierarchy problem similar to that of ref. 8 .

Other solutions obtained with two transverse dimensions include a generalization of the original 5d domain wall setup to the case of parallel brane sources [9], and the case of global string defects [10, 11, 12]. Furthermore, a class of radially symmetric solutions was considered in [13, 14].

Let us begin with the details of our solution. In $6 \mathrm{~d}$ the Einstein equations with a bulk cosmological constant $\Lambda$ and stress-energy tensor $T_{A B}$ are

$$
R_{A B}-\frac{1}{2} g_{A B} R=\frac{1}{M_{6}^{4}}\left(\Lambda g_{A B}+T_{A B}\right)
$$

where $M_{6}$ is the six-dimensional reduced Planck scale. We will assume that there exists a solution that respects $4 \mathrm{~d}$ Poincare invarance. A six-dimensional metric satisfying this ansatz is

$$
d s^{2}=\sigma(\rho) g_{\mu \nu} d x^{\mu} d x^{\nu}-d \rho^{2}-\gamma(\rho) d \theta^{2},
$$

where the metric signature is $(+,-,-,-)$. For the two extra spatial dimensions we have introduced polar coordinates $(\rho, \theta)$, where $0 \leq \rho<\infty$ and $0 \leq \theta<2 \pi$. With our metric ansatz (4), the general expression for the four-dimensional reduced Planck scale, $M_{P}$ expressed in terms of $M_{6}$ is

$$
M_{P}^{2}=2 \pi M_{6}^{4} \int_{0}^{\infty} d \rho \sigma \sqrt{\gamma}
$$

The nonzero components of the stress-energy tensor $T_{B}^{A}$ are assumed to be

$$
T_{\nu}^{\mu}=\delta_{\nu}^{\mu} f_{0}(\rho), \quad T_{\rho}^{\rho}=f_{\rho}(\rho), \quad \text { and } \quad T_{\theta}^{\theta}=f_{\theta}(\rho)
$$

where we have introduced three source functions $f_{0}, f_{\rho}$ and $f_{\theta}$, which depend only on the radial coordinate $\rho$. Using the cylindrically symmetric metric ansatz (4) and the stress energy tensor (6), the Einstein equations become

$$
\begin{aligned}
\frac{3}{2} \frac{\sigma^{\prime \prime}}{\sigma}+\frac{3}{4} \frac{\sigma^{\prime}}{\sigma} \frac{\gamma^{\prime}}{\gamma}-\frac{1}{4} \frac{\gamma^{\prime 2}}{\gamma^{2}}+\frac{1}{2} \frac{\gamma^{\prime \prime}}{\gamma} & =-\frac{1}{M_{6}^{4}}\left(\Lambda+f_{0}(\rho)\right)+\frac{1}{M_{P}^{2}} \frac{\Lambda_{p h y s}}{\sigma} \\
\frac{3}{2} \frac{\sigma^{\prime 2}}{\sigma^{2}}+\frac{\sigma^{\prime}}{\sigma} \frac{\gamma^{\prime}}{\gamma} & =-\frac{1}{M_{6}^{4}}\left(\Lambda+f_{\rho}(\rho)\right)+\frac{1}{M_{P}^{2}} \frac{2 \Lambda_{p h y s}}{\sigma} \\
2 \frac{\sigma^{\prime \prime}}{\sigma}+\frac{1}{2} \frac{\sigma^{\prime 2}}{\sigma^{2}} & =-\frac{1}{M_{6}^{4}}\left(\Lambda+f_{\theta}(\rho)\right)+\frac{1}{M_{P}^{2}} \frac{2 \Lambda_{p h y s}}{\sigma}
\end{aligned}
$$

where the 'denotes differentiation $d / d \rho$. The constant $\Lambda_{\text {phys }}$ represents the physical fourdimensional cosmological constant, where

$$
R_{\mu \nu}^{(4)}-\frac{1}{2} g_{\mu \nu} R^{(4)}=\frac{1}{M_{P}^{2}} \Lambda_{p h y s} g_{\mu \nu} .
$$


By eliminating two of the equations in (7), the sources can be related by the equation

$$
f_{\rho}^{\prime}=2 \frac{\sigma^{\prime}}{\sigma}\left(f_{0}-f_{\rho}\right)+\frac{1}{2} \frac{\gamma^{\prime}}{\gamma}\left(f_{\theta}-f_{\rho}\right)
$$

In the absence of source terms, the discussion of solutions to this coupled system of differential equations for arbitrary values of $\Lambda_{\text {phys }}$ and $\Lambda>0$ can be found in [2]. However, the case $\Lambda<0$ was not considered there because the vacuum solutions lead to noncompact transverse spaces, and therefore using (5), one cannot obtain a finite value of the Planck scale. Here we propose adding singular source terms in order to obtain a transverse space with finite volume (which leads to a finite four-dimensional Planck scale). Thus, the system of equations (7) and (9) describes the generalization of the setup considered in [2], to the case where source terms are included. Similar equations of motion in the global string context, were also considered in Refs. [10, 11, 12].

Specifically, we will assume that there is a 3-brane at the origin $\rho=0$ which is a fourdimensional local string-like topological defect in the six-dimensional spacetime, and has a nonzero stress-energy tensor $T_{B}^{A}$ parametrized by (6). For example, one may think of the Nielsen-Olesen string solution in the $6 \mathrm{~d}$ Abelian Higgs model. The source functions describe a continuous matter distribution within the core of radius $\epsilon$ and vanish for $\rho>\epsilon$. At the origin we will require that our solution satisfies the boundary conditions

$$
\left.\sigma^{\prime}\right|_{\rho=0}=0,\left.\quad(\sqrt{\gamma})^{\prime}\right|_{\rho=0}=1 \text { and }\left.\gamma\right|_{\rho=0}=0
$$

We have set $\sigma(0)=A$, where $A$ is a constant. Following [15, we can integrate over the disk of small radius $\epsilon$ containing the 3 -brane, and define various components of the string tension per unit length as

$$
\mu_{i}=\int_{0}^{\epsilon} d \rho \sigma^{2} \sqrt{\gamma} f_{i}(\rho) .
$$

where $i=0, \rho, \theta$. Using the system of equations (7) we obtain the following boundary conditions

$$
\left.\sigma \sigma^{\prime} \sqrt{\gamma}\right|_{0} ^{\epsilon}=-\frac{1}{2 M_{6}^{4}}\left(\mu_{\rho}+\mu_{\theta}\right)
$$

and

$$
\left.\sigma^{2}(\sqrt{\gamma})^{\prime}\right|_{0} ^{\epsilon}=-\frac{1}{M_{6}^{4}}\left(\mu_{0}+\frac{1}{4} \mu_{\rho}-\frac{3}{4} \mu_{\theta}\right),
$$

where it is understood that the limit $\epsilon \rightarrow 0$ is taken. By analogy with string defects in four dimensions, $\mu_{\rho}+\mu_{\theta}$ can be referred to as the Tolman mass (per unit length) [16]. Its nonzero value in four dimensions gives rise to the Melvin branch for local string defects [17]. Similarly, the analogous equation of (13) in four dimensions is related to the string angular deficit [17]. Thus, with these general conditions, any metric solution to the Einstein equations with sources will lead to nontrivial relationships between the components of the string tension per unit length. 
Let us now restrict to the case where the four-dimensional cosmological constant $\Lambda_{\text {phys }}=0$, and look for a solution outside the core $(\rho>\epsilon)$ of the form

$$
\sigma(\rho)=e^{-c \rho} .
$$

Note that we have chosen the arbitrary integration constant, which corresponds to an overall rescaling of the coordinates $x^{\mu}$, such that $\lim _{\epsilon \rightarrow 0} \sigma(\epsilon)=1$. Then, a solution to the coupled set of equations (7) can be found with $\gamma(\rho)=R_{0}^{2} \sigma(\rho)$ and

$$
c=\sqrt{\frac{2}{5} \frac{(-\Lambda)}{M_{6}^{4}}},
$$

where $R_{0}$ is an arbitrary length scale that can be fixed from eqs. (12) and (13). Clearly, the negative exponential solution (14) requires that $\Lambda<0$. If we now demand that the solution (15) is consistent with the boundary conditions (12) and (13), the components of the string tension per unit length must satisfy

$$
\mu_{0}=\mu_{\theta}+A^{2} M_{6}^{4}
$$

where $\mu_{\rho}$ remains undetermined. In fact choosing $\mu_{\rho}=0$ gives

$$
\mu_{\theta}=2 R_{0} M_{6}^{4} c .
$$

Thus, as long as sources are introduced at the origin $\rho=0$ satisfying (16), we obtain a flat Poincare invariant solution in four dimensions. Since the solution is already valid for $\Lambda_{\text {phys }}=0$, there is no need to tune the brane tension to the bulk cosmological constant, $\Lambda$ as in the case [7]. However, there is still a tuning in order to satisfy (16).

Having found a solution with a finite volume transverse space the four-dimensional reduced Planck scale now becomes

$$
M_{P}^{2}=2 \pi R_{0} M_{6}^{4} \int_{0}^{\infty} d \rho \sigma^{3 / 2}=\frac{5 \pi}{3} \frac{\mu_{\theta}}{-\Lambda} M_{6}^{4},
$$

where we have used the relation (17). The inequality $M_{6} \ll M_{P}$ is possible by adjusting the string tension or the bulk cosmological constant, and thus could lead to a solution of the gauge hierarchy problem along the lines of [8] .

In order to see that gravity is only localized on the 3-brane, let us now consider the equations of motion for the linearized metric fluctuations. We will only concentrate on the spin-2 modes and neglect the scalar modes, which needs to be taken into account together with the bending of the brane [18]. The vector modes are massive as follows from a simple modification of the results in Ref. [19]. For a fluctuation of the form $h_{\mu \nu}(x, z)=\Phi(z) h_{\mu \nu}(x)$ where $z=(\rho, \theta)$ and $\partial^{2} h_{\mu \nu}(x)=m_{0}^{2} h_{\mu \nu}(x)$ we can separate the variables by defining $\Phi(z)=\sum_{l m} \phi_{m}(\rho) e^{i l \theta}$. The radial modes satisfy the equation [19]

$$
-\frac{1}{\sigma \sqrt{\gamma}} \partial_{\rho}\left[\sigma^{2} \sqrt{\gamma} \partial_{\rho} \phi_{m}\right]=m^{2} \phi_{m}
$$


where $m_{0}^{2}=m^{2}+l^{2} / R_{0}^{2}$ contains the contributions from the orbital angular momentum $l$. The differential operator (19) is self-adjoint provided that we impose the boundary conditions

$$
\phi_{m}^{\prime}(0)=\phi_{m}^{\prime}(\infty)=0,
$$

where the modes $\phi_{m}$ satisfy the orthonormal condition

$$
\int_{0}^{\infty} d \rho \sigma \sqrt{\gamma} \phi_{m}^{*} \phi_{n}=\delta_{m n} .
$$

Using the specific solution (15), the differential operator (19) becomes

$$
\phi_{m}^{\prime \prime}-\frac{5}{2} c \phi_{m}^{\prime}+m^{2} e^{c \rho} \phi_{m}=0 .
$$

This equation is the same as that obtained for the $5 \mathrm{~d}$ domain wall solution [7], except that the coefficient of the the first-derivative term is 2 instead of $5 / 2$. This difference is due to the extra spatial coordinate in the transverse space. When $m=0$ we clearly see that $\phi_{0}(\rho)=$ constant is a solution. Since the modes satisfy the orthonormal condition

$$
R_{0} \int_{0}^{\infty} d \rho e^{-\frac{3}{2} c \rho} \phi_{m}^{*} \phi_{n}=\delta_{m n},
$$

a wavefunction in flat space can be defined as

$$
\psi_{m}=e^{-\frac{3}{4} c \rho} \phi_{m} .
$$

Thus the zero-mode wavefunction becomes

$$
\psi_{0}(\rho)=\sqrt{\frac{3 c}{2 R_{0}}} e^{-\frac{3}{4} c \rho},
$$

which shows that the zero-mode tensor fluctuation is localized near the origin $\rho=0$ and is normalizable.

The contribution from the nonzero modes will modify Newton's law on the 3-brane. In order to calculate this contribution we need to obtain the wavefunction for the nonzero modes at the origin. The nonzero mass eigenvalues can be obtained by imposing the boundary conditions (20) on the solutions of the differential equation (22). The solutions of (22) are

$$
\phi_{m}(\rho)=e^{\frac{5}{4} c \rho}\left[C_{1} J_{5 / 2}\left(\frac{2 m}{c} e^{\frac{c}{2} \rho}\right)+C_{2} Y_{5 / 2}\left(\frac{2 m}{c} e^{\frac{c}{2} \rho}\right)\right],
$$

where $C_{1}, C_{2}$ are constants and $J_{5 / 2}, Y_{5 / 2}$ are Bessel functions which can be expressed in terms of elementary functions. In the limit that $\rho \rightarrow \infty$, the solutions for nonzero $m$ grow exponentially. One way to regulate this behaviour is to introduce a finite radial distance cutoff $\rho_{\max }$. Then imposing the boundary conditions (20) at $\rho=\rho_{\max }$ (instead of $\rho=\infty$ ) will lead to a discrete mass spectrum, where for sufficiently large integer $n$ we obtain

$$
m_{n} \simeq c\left(n-\frac{1}{2}\right) \frac{\pi}{2} e^{-\frac{c}{2} \rho_{\max }} .
$$


With this discrete mass spectrum we find that in the limit of vanishing mass $m_{n}$,

$$
\phi_{m_{n}}^{2}(0)=\frac{4}{c R_{0}} m_{n}^{2} e^{-\frac{c}{2} \rho_{\max }} .
$$

On the 3-brane the gravitational potential between two point masses $m_{1}$ and $m_{2}$, will receive a contribution from the discrete nonzero modes given by

$$
\Delta V(r) \simeq G_{N} \frac{m_{1} m_{2}}{r} \sum_{n} e^{-m_{n} r} \frac{8}{3 c^{2}} m_{n}^{2} e^{-\frac{c}{2} \rho_{\max }}
$$

where $G_{N}$ is Newton's constant. In the limit that $\rho_{\max } \rightarrow \infty$, the spectrum becomes continuous and the discrete sum is converted into an integral. Thus the contribution to the gravitational potential becomes

$$
\begin{aligned}
\Delta V(r) & \simeq \frac{16 G_{N}}{3 \pi c^{3}} \frac{m_{1} m_{2}}{r} \int_{0}^{\infty} d m m^{2} e^{-m r} \\
& =\frac{32 G_{N}}{3 \pi c^{3}} \frac{m_{1} m_{2}}{r^{4}}
\end{aligned}
$$

Thus we see that the correction to Newton's law from the bulk continuum states grows like $1 / r^{3}$. This correction is more suppressed than in $5 \mathrm{~d}$, because now the gravitational field of the bulk continuum modes spreads out in one extra dimension and so their effect on the 3-brane is weaker.

Some remarks are now in order:

(i) If different components of the brane tension do not satisfy equation (16), a more general solution to the system of equations (7) can be found along the lines of ref. [2]. Using the parametrisation $\sigma=z^{4 / 5}$, and $\gamma=\alpha^{2}\left(z^{\prime}\right)^{2} z^{-6 / 5}$ (with $\alpha=4 R_{0} /(5 c)$ ) the general solution can be written as

$$
z(\rho)=\exp \left(-\frac{5}{4} c \rho\right)+2 \beta \sinh \left(-\frac{5}{4} c \rho\right)
$$

where $\beta=0$ corresponds to the case (14). The general condition for the brane tension components now becomes

$$
\mu_{0}-\mu_{\theta}=\beta(\beta+1)\left(\frac{3}{2} \mu_{\theta}-\frac{5}{2} \mu_{\rho}-4 \mu_{0}\right)+(1+2 \beta)^{2} A^{2} M_{6}^{4} .
$$

The choice of $\beta<0$ does not lead to any compactification because $\sigma$ diverges at large $\rho$. However, $\beta>0$ leads to non-compact spaces defined for a finite interval $0<\rho<\frac{2}{5 c} \log \left(\frac{1+\beta}{\beta}\right)$ of the type discussed in [2] that may be used as a description of four-dimensional space.

(ii) The metric solution that we have found can also be written in the form

$$
d s^{2}=z^{2} g_{\mu \nu} d x^{\mu} d x^{\nu}-z^{2} R_{0}^{2} d \theta^{2}-\frac{4}{c^{2} z^{2}} d z^{2}
$$


where $z=\exp \left(-\frac{c}{2} \rho\right)$. In this way we see that the origin $\rho=0$ is now mapped to $z=1$. The singular source is spread around the circumference of a disk of radius $R_{0}$. This suggests that the 3-brane at the origin $\rho=0$ can be interpreted as a wrapped 4-brane where all angular points, $\theta$ are identified. In other words, denoting the wrapped 4 -brane by $\mathcal{M}_{4}$, then the 3 -brane corresponds to $\mathcal{M}_{4} / S^{1}$. While we have given the explicit solution in six dimensions, our solution can be generalized and presumably similar solutions exist at the core of topological defects in higher dimensions where for $n \geq 2$ transverse dimensions the 3-brane can be identified with

$\mathcal{M}_{n+2} / S^{n-1}$, where $\mathcal{M}_{n+2}$ has $n-1$ coordinates spherically wrapped. Again, the corrections to $4 \mathrm{~d}$ gravity on the 3-brane are expected to be small since the bulk continuum modes live in a higher-dimensional space and by Gauss's law the effects on the 3-brane are suppressed.

(iii) It is also interesting to study whether our solution (or its generalization in higher dimensions) can be realized in an effective supergravity theory. This would be one step towards embedding the scenario in string theory.

Acknowledgments: We wish to thank S. Dubovsky and P. Tinyakov for helpful discussions. This work was supported by the FNRS, contract no. 21-55560.98.

\section{References}

[1] Modern Kaluza-Klein Theories, eds. T. Appelquist, A. Chodos and P. G. Freund, (AddisonWesley, 1987).

[2] V. A. Rubakov and M. E. Shaposhnikov, Phys. Lett. B125 (1983) 139.

[3] V. A. Rubakov and M. E. Shaposhnikov, Phys. Lett. B125 (1983) 136.

[4] K. Akama, in Proceedings of the Symposium on Gauge Theory and Gravitation, Nara, Japan, eds. K. Kikkawa, N. Nakanishi and H. Nariai (Springer-Verlag, 1983), hepth/0001113.

[5] M. Visser, Phys. Lett. B159 (1985) 22 hep-th/9910093.

[6] J. Polchinski, Phys. Rev. Lett. 75 (1995) 4724 hep-th/9510017.

[7] L. Randall and R. Sundrum, Phys. Rev. Lett. 83 (1999) 4690 hep-th/9906064.

[8] N. Arkani-Hamed, S. Dimopoulos and G. Dvali, Phys. Lett. B429 (1998) 263 hepph/9803315.

[9] A. Chodos and E. Poppitz, Phys. Lett. B471 (1999) 119 [hep-th/9909199].

[10] A. G. Cohen and D. B. Kaplan, Phys. Lett. B470 (1999) 52 hep-th/9910132.

[11] R. Gregory, Phys. Rev. Lett. 84 (2000) 2564 hep-th/9911015.

[12] I. Olasagasti and A. Vilenkin, hep-th/0003300. 
[13] C. Csaki, J. Erlich, T. J. Hollowood and Y. Shirman, hep-th/0001033.

[14] J. Chen, M. A. Luty and E. Ponton, hep-th/0003067.

[15] V. P. Frolov, W. Israel and W. G. Unruh, Phys. Rev. D39 (1989) 1084.

[16] M. Christensen, A. L. Larsen and Y. Verbin, Phys. Rev. D60 (1999) 125012 grqc/9904049].

[17] Y. Verbin, Phys. Rev. D59 (1999) 105015 [hep-th/9809002].

[18] J. Garriga and T. Tanaka, hep-th/9911055; S. B. Giddings, E. Katz and L. Randall, JHEP 0003 (2000) 023 hep-th/0002091.

[19] G. V. Lavrelashvili and P. G. Tinyakov, Sov. J. Nucl. Phys. 41 (1985) 172. 